\title{
Evolution by selection, recombination, and gene duplication in MHC class I genes of two Rhacophoridae species
}

\author{
Mian Zhao, Yongzhen Wang, Hang Shen, Chenliang Li, Cheng Chen, Zhenhua Luo and Hua Wu*
}

\begin{abstract}
Background: Comparison of major histocompatibility complex (MHC) genes across vertebrate species can reveal molecular mechanisms underlying the evolution of adaptive immunity-related proteins. As the first terrestrial tetrapods, amphibians deserve special attention because of their exposure to probably increased spectrum of microorganisms compared with ancestral aquatic fishes. Knowledge regarding the evolutionary patterns and mechanisms associated with amphibian MHC genes remains limited. The goal of the present study was to isolate MHC class I genes from two Rhacophoridae species (Rhacophorus omeimontis and Polypedates megacephalus) and examine their evolution.

Results: We identified $27 \mathrm{MHC}$ class I alleles spanning the region from exon 2 to 4 in 38 tree frogs. The available evidence suggests that these 27 sequences all belong to classical MHC class I (MHC la) genes. Although several anuran species only display one MHC class la locus, at least two or three loci were observed in P. megacephalus and $R$. omeimontis, indicating that the number of MHC class la loci varies among anuran species. Recombination events, which mainly involve the entire exons, played an important role in shaping the genetic diversity of the $27 \mathrm{MHC}$ class la alleles. In addition, signals of positive selection were found in Rhacophoridae MHC class la genes. Amino acid sites strongly suggested by program to be under positive selection basically accorded with the putative antigen binding sites deduced from crystal structure of human HLA. Phylogenetic relationships among MHC class I alleles revealed the presence of trans-species polymorphisms.

Conclusions: In the two Rhacophoridae species (1) there are two or three MHC class la loci; (2) recombination mainly occurs between the entire exons of MHC class la genes; (3) balancing selection, gene duplication and recombination all contribute to the diversity of MHC class la genes. These findings broaden our knowledge on the evolution of amphibian MHC systems.
\end{abstract}

Keywords: Major histocompatibility complex, Evolution, Rhacophorus omeimontis, Polypedates megacephalus

\section{Background}

Genes of the major histocompatibility complex (MHC) encode cell surface glycoproteins that present self and foreign (e.g., pathogens) peptides to $\mathrm{T}$ lymphocytes to trigger the appropriate adaptive immune response. These glycoproteins can be divided into two major subgroups: class I and class II MHC molecules, which recognize intracellular and extracellular antigens, respectively. Class I molecules consist of a heavy chain ( $\alpha$ chain) and a microglobulin chain

\footnotetext{
*Correspondence: wuhua@mail.ccnu.edu.cn

Molecular and Behavioural Ecology Research Group, College of Life Sciences, Central China Normal University, 152 Luoyulu, Hongshan District, Wuhan 430079, China
}

\section{Biomed Central

(c) 2013 Zhao et al.; licensee BioMed Central Ltd. This is an Open Access article distributed under the terms of the Creative Commons Attribution License (http://creativecommons.org/licenses/by/2.0), which permits unrestricted use, distribution, and reproduction in any medium, provided the original work is properly cited.
( $\beta_{2} \mathrm{~m}$ chain). The heavy chain has a cytoplasmic region, a trans-membrane region, and three extracellular domains designated $\alpha 1, \alpha 2$, and $\alpha 3$ that are encoded by exons $2-4$ of the MHC class I genes. The amino acid sites that recognize and bind antigen (antigen binding sites, $\mathrm{ABS}$ ) are located in domains $\alpha 1$ and $\alpha 2$ [1,2]. Certain MHC class I genes that display a very high level of genetic diversity $[3,4]$ are called classical MHC class I genes or MHC class Ia genes [2]. In contrast, non-classical MHC class I (MHC class Ib) genes possess fewer polymorphisms, are expressed at lower levels in fewer tissues, and perform different functions despite having similar sequences $[5,6]$. 
The high level of MHC class I gene polymorphisms is not only due to increased nucleotide and gene diversity [7-9] but also to increased variations on locus numbers [10-12]. More than one MHC class I locus has been observed in many vertebrate categories, and the number of loci varies among species $[10,11,13,14]$. These polymorphisms are thought to correspond to the function of the $\mathrm{MHC}$ because a greater number of polymorphic $\mathrm{MHC}$ proteins provide organisms protection against a larger spectrum of pathogens and thus better fitness. In fact, this hypothesis is supported by the correlation between MHC diversity and disease susceptibility observed in many vertebrates [15-17]. Thus, the MHC class I gene is an appropriate genetic marker to study adaptive evolution [17-19].

The evolutionary mechanisms contributing to the diversity of MHC class I genes are of significant interest in the study of MHC evolution. Three main mechanisms have been proposed: (1) balancing selection [19-22], (2) recombination [23-26] and (3) gene duplication [12,13]. (1) An excess of non-synonymous over synonymous substitutions, especially on ABS, is typically detected in $\mathrm{MHC}$ class I genes, supporting the occurrence of positive selection $[4,27,28]$. Due to the coexistence of many relatively frequent alleles at an individual locus, the type of selection that acts on $\mathrm{MHC}$ genes is designated balancing selection. Under balancing selection, MHC alleles can be maintained for millions of years; they are passed from ancestral to descendant species such that closely related species with a relatively recent common ancestor present identical or similar alleles. The phylogenetic tree constructed using these alleles revealed that sequences from the same species could have more distant relationships compared with those from different species. This phenomenon is called trans-species polymorphism and is commonly observed in MHC genes [2,29,30]. (2) In many studies, recombination has been shown to be an important evolutionary mechanism responsible for the extremely high level of genetic diversity of MHC class I genes [23-26]. Two types of recombination patterns have been found. One is exon shuffling, which is characterized by recombination between entire exon regions with breakpoints in intronic regions [31]. This type of recombination is common in teleosts $[25,32,33]$, likely due to the presence of repetitive elements in the long introns of teleosts' MHC genes [33]. The other recombination pattern is mainly observed in mammals and involves exchanges of small fragments of MHC class I genes [34]. (3) Variations in the number of alleles or loci appear when some of the duplicated genes are maintained in the genome for long periods while others become dysfunctional due to deleterious mutations. The mechanism underlying this type of MHC evolution is called the birth-and-death model [35]. It plays an important role in driving the evolution of $\mathrm{MHC}$ genes in many vertebrates $[12,13,26]$.
Knowledge on the evolution of MHC class I genes was obtained mainly from studies of mammals, birds, and fish $[4,10,18]$. Amphibians theoretically deserve special attention while studying $\mathrm{MHC}$ evolution because they are the first terrestrial vertebrates, probably facing a very different spectrum and diversity of microorganisms. However, relatively few studies have focused on the evolution of amphibian MHC class I genes, and those few studies have involved a limited number of amphibian species, mainly Ambystoma mexicanum in the Caudata [36] and two closely related Pipidae species (Xenopus lavies and X. (Silurana) tropicalis) in the Anura [37-39]. These studies revealed that (1) Urodela (Ambystoma) have multiple MHC class I loci [36], whereas the Anura (Pipidae and Ranidae) have only one [37-41]; it is possible that the number of MHC class I loci increased after the Urodela and Anura but before the Pipidae and Ranidae diverged [40]. (2) Both balancing selection and recombination play an important role in the evolution of MHC class I genes [37,38]; recombination occurred among entire exons, at least in X. lavies [37]. (3) Transspecies polymorphisms are common in amphibian $\mathrm{MHC}$ class I genes [38]. It is unknown whether the rules revealed by the evolution of a limited number of species can apply to all amphibians. The extent to which Xenopus species represent the Anura is limited because the two Xenopus species in Pipidae group diverged early from the ancestral anurans $[42,43]$ and live a predominantly aquatic life that differs from the lifestyle of most anurans. A recent study examining six non-model anuran species (family Hylidae: Agalychnis callidryas, Smilisca phaeota; family Centrolenidae: Espadarana prosoblepon; family Ranidae: Lithobates catesbeianus, L. clamitans, L. yavapaiensis) revealed more complex patterns and mechanisms in the evolution of MHC class I genes [44], which suggests that a greater number of species must be assessed to obtain a more systematic understanding of the evolution of amphibian MHC class I genes. Consequently, studies examining $\mathrm{MHC}$ evolution of a greater number of anuran species may be pivotal in better understanding the evolutionary process of acquired immunity in vertebrates.

The Rhacophoridae family includes arboreal species likely facing multifarious microorganisms due to the moist habitat. It belongs to the Ranoidae/Natatanura clade; the Ranidae family is its sister taxon [42]. Here, we selected two Rhacophoridae species, Rhacophorus omeimontis and Polypedates megacephalus, to conduct the following studies: (1) designing primers to isolate MHC class I genes from the two species; (2) assessing phylogenetic status of the newly isolated genes; (3) identifying the number of MHC class I loci; and (4) screening for signals of natural selection and recombination. The results of this study will provide insight into the evolution of anuran MHC class I genes. 


\section{Methods}

\section{Sampling and RNA extraction}

We sampled 11 P. megacephalus (all from Sangzhi County, Hunan Province) and 27 R. omeimontis (12 from Sangzhi County, Hunan Province and 15 from Baoxing County, Sichuan Province). Total RNA was extracted from liver tissues using TRIzol according to the manufacturer's recommendations (Invitrogen). All experiments involving live tree frogs were approved by the Animal Ethics Committee at Central China Normal University.

\section{Primer design}

To design appropriate primers to amplify MHC class I genes from our samples, we searched NCBI for published amphibian MHC class I sequences. MHC class I alleles from $A$. mexicanum (AMU83137), X. laevis/gilli (AF185583), Rana temporaria (FJ385652), R. pipiens (AF185587), and X. ruwenzoriensis (AF497528) were then compiled and aligned. Based on conserved regions of these sequences, we designed the following primers using Primer3 [45]: forward primer 5'- CTGCGSWAYTATKABACWGCAGTCTC -3'; reverse primer 5'- TYCAGRCTGCTGTGSTCCACAT -3'.

\section{DNA amplification and cloning}

First-strand complementary DNA (cDNA) was synthesized from total RNA using reverse transcriptase M-MLV (TaKaRa) with oligo $(\mathrm{dT})_{18}$ primers and then used as a template for the polymerase chain reaction (PCR) to amplify MHC class I exons 2-4 from our samples. The PCR products were purified using the kit (Promega) and then cloned into the pMD19-T vector (TaKaRa). Recombinant DNA was transformed into TOP-10 Escherichia coli cells, which were then plated onto Luria-Bertani (LB) agar and grown overnight at $37^{\circ} \mathrm{C}$. Clones were randomly selected to screen for the presence of the inserted DNA fragments by PCR and agarose gel electrophoresis. Approximately 20 colonies with positive transformants were selected for each sample, and each transformant was cultivated separately in $\mathrm{LB}$ broth at $37^{\circ} \mathrm{C}$ for $12 \mathrm{~h}$. The positive clones were sequenced.

Noticeably, we assessed our newly designed primers before amplifying MHC class I genes from large amounts of samples. First, only one sample was randomly selected for RNA extraction, cDNA synthesis, and MHC class I gene amplification, as described above. The sequences of 5 positive clones were blasted on NCBI. These sequences were found to have a very high similarity $(>80 \%)$ with the published anuran MHC class I genes, which suggests that our primer design was successful.

\section{Sequence alignment and allele identification}

ABI trace files were examined and edited using DNASTAR4. The sequences were then aligned using ClustalW equipped in MEGA5 [46]. Only those sequences that were present in more than one individual were considered alleles and were included in the following analyses.

The newly obtained alleles were translated into amino acid sequences and aligned with human HLA chains with a published three-dimensional structure $[47,48]$. The structures permitted the identification of key amino acid sites that are likely involved in peptide binding and disulfide bond formation.

\section{Statistical analysis}

The average pairwise nucleotide distances (Kimura-2-parameter model, K2P) and the Poisson-corrected amino acid distances were computed using MEGA5 [46]. Standard errors of the estimates were obtained via 1000 bootstrap replicates.

Several methods were used to detect recombination in our datasets. One method was conducted using the online program GARD (genetic algorithm recombination detection; [49]) at the Datamonkey website (http://www. datamonkey.org/). The other methods, including RDP [50], BOOTSCAN [51,52], GENECONV [53], MAXCHI [54,55], CHIMAERA [55], SISCAN [56], and 3SEQ [57], are all implemented in the program suite Recombination Detection Program version 3 (RDP3; [58]). To minimize the false-positive error rate, the highest acceptable $P$ value for inferring recombination events was set at 0.000005 with a window size of 20 nucleotides, and only those breakpoints that were identified using at least four methods were considered valid.

Phylogenetic relationships were constructed using the anuran MHC class I alleles determined herein and those available in NCBI. The compiled, published data included MHC class Ia genes from A. callidryas, S. phaeota, E. prosoblepon, L. catesbeianus, L. clamitans, L. yavapaiensis, and $R$. pipiens, as well as MHC class Ia and Ib genes from $X$. tropicalis and $X$. laevis. To determine the general phylogenetic status of the newly isolated MHC class I genes, MEGA5 was used to construct a neighbor-joining (NJ) tree using the exon 2-4 region of the MHC class I genes [46]. Considering that recombination events can hinder the identification of true phylogenetic relationships, the sequences were then partitioned as individual exons to infer more accurate phylogenetic trees of anuran species MHC class I genes based on the recombination test results. For this procedure, approximately three alleles from each species with available MHC class I gene sequences in NCBI were randomly selected as representatives for inclusion in the tree construction. Selection of the best-fitting models of nucleotide substitution was conducted based on Akaike Information Criterion (AIC) in jModelTest 0.1 $[59,60]$. Analyses revealed that TPM2uf $+\mathrm{G}, \operatorname{TrN}+\mathrm{G}$, and TPM3 + I + G were the most appropriate models for exon 2 , exon 3, and exon 4, respectively. Bayesian inference 




Figure 1 Phylogenetic relationships of anuran MHC class la and lb genes. The neighbor-joining tree was constructed with MEGA5 using all available anuran MHC class I genes with a sufficient length. Bootstrap values are indicated above the branches. Sample names are not shown due to the large size of the tree. Rather, the families to which these samples belong are denoted with different colors. The blue horizontal line separates MHC class la genes (above the line) and MHC class Ib genes (below the line). Alleles in this tree include the following: the 27 alleles isolated in the present study (family Rhacophoridae); MHC class la alleles from the family Hylidae (19 alleles from A. callidryas: JQ679312-JQ679330; 11 alleles from S. phaeota: JQ679380-JQ679390, [44]); from family Centrolenidae (12 alleles from E. prosoblepon: JQ679331-JQ679342, [44]); from family Ranidae (12 alleles from R. catesbeiana: JQ679343-JQ679354; 16 alleles from R. clamitans: JQ679355-JQ679370; 9 alleles from R. yavapaiensis: JQ679371-JQ679379, [44]; 2 alleles from R. pipiens: AF185587-AF185588, [72]); from family Pipidae ( 5 alleles from X. tropicalis: BC167634, BC161748, BC154904, [73]; NM_001112910, NM_001113065, [74]); 2 alleles from X. laevis: (NM_001085732, [72]; NM_001086402, [73]); and MHC class Ib alleles from family Pipidae (6 alleles from X. laevis: L20726, L20730, L20732, [6]; FJ589642- FJ589643, [75]; NM_001135072, [72]; 2 MHC Ib alleles from X. tropicalis: NM_001037273, NM_001247995, [72]).

trees were then constructed using MrBayes 3.2 [61]. Two independent runs of four Metropolis coupled Markov chain Monte Carlo (MCMC) simulations (three of them 'heated', temperature $=0.20$ ) were performed for millions of generations until the standard deviation of the split frequencies was $<0.01$. The first $25 \%$ of the sampled trees from the cold chain were discarded by default. The remaining trees were then used to compute the majorityrule consensus tree and to calculate the posterior probability of each bipartition. In addition, neighbor-joining trees of individual exons were constructed using the matrix of nucleotide distances through MEGA5 [46]. Support for the nodes in the obtained tree was estimated via 1000 bootstrap replicates.

Recombination leads to false inferences of positive selection. Fortunately, partitioning strategy considering separate unrecombinant segments based on deduced recombination breakpoints can largely reduce false positive to acceptable level [62]. We used three approaches all with partitioning pre-analyses to test whether positive selection shaped the evolution pattern of the newly isolated MHC class I sequences. First, the presence of 
positive selection signals was tested using the Codeml subroutine included in PAML version $4[63,64]$. Maximum likelihood estimations of $\omega(\omega=\mathrm{dN} / \mathrm{dS}$, that is, the ratio of non-synonymous/synonymous substitution rates) among codons were generated using this procedure $[65,66]$. Six different models corresponding to different distribution patterns of $\omega$ were tested in our data: M0 (one $\omega$ ), M1a (nearly neutral), M2a (positive selection), M3 (discrete); M7 (nearly neutral with the beta distribution approximating $\omega$ variation; M8 (positive selection with the beta distribution approximating $\omega$ variation) [65-67]. Three likelihood ratio tests (LRTs) were then performed to compare the nested models (M0 vs. M3, M1a vs. M2a, and M7 vs. M8) to test the presence of positive selection in models M2a, M3, and M8. Positively selected sites were identified by Bayes empirical Bayes (BEB) method in models M2a and M8 [67]. However, another two methods for detecting selection signals were also applied considering that the power of Codeml would be affected by the accuracy of the inferred phylogenetic trees [68]. Both methods (FEL and MEME) [69-71] were implemented at the Datamonkey website (http://www.datamonkey.org). A more accurate understanding of the sites affected by positive selection can be obtained by combining the results from all of the present analyses.

\section{Results}

\section{Allele characterization}

Using the techniques described in the Methods, we obtained 757 sequences and finally identified 27 putative MHC class I alleles. These alleles span a portion of exon 2 and of exon 4 and the entire region of exon 3, with a length of 732 nucleotides or 735 nucleotides. Length variation resulting in an indel of one amino acid codon is very common in amphibian species [40,72,73]. Additionally, none of these sequences displayed stop codons. As a result, all of the 27 alleles could be successfully translated into peptides. Comparisons of these peptides with human HLA amino acid sequences revealed that the four pivotal amino acid sites that form disulfide bridges (Cys101Cys164 and Cys203-Cys259 in human HLA) were conserved in our focal sequences $[47,48]$. This finding indicated that all of the identified alleles were functional. Next, we constructed a phylogenetic tree using the 27 alleles and all of the published MHC class I genes from anurans with comparable coverage (see Methods) to resolve their phylogenetic status. We found that MHC class Ia genes and MHC class Ib genes clustered separately, and all of the 27 Rhacophoridae alleles belonged to the MHC class Ia gene cluster (Figure 1). This result further suggested that all of the 27 alleles were located at the MHC class Ia locus. We deposited these alleles in GenBank under accession numbers KC261637-KC261663.

\section{Number of loci}

We identified 7 alleles in 11 P. megacephalus, 6 alleles in $12 R$. omeimontis from Hunan, and 14 alleles in $15 R$. omeimontis from Sichuan. None of these alleles was shared among the three populations. At most three, three, and six alleles were observed respectively in each $P$. megacephalus, $R$. omeimontis from Hunan, and $R$. omeimontis from Sichuan. As $P$. megacephalus and $R$. omeimontis are both diploid [76,77], we concluded that they possess 2 to 3 MHC class Ia loci (Table 1). This result is inconsistent with the findings of previous studies examining two Xenopus species and two Rana species, from which only one MHC class Ia locus was observed $[5,39,40,72,78]$. However, it is in accordance with the results of a recent study of 6 nonmodel anuran species with 2 or $3 \mathrm{MHC}$ class Ia loci [44]. Noticeably, the actual number of loci was probably larger than our prediction because we identified the alleles using a very conservative strategy, and the locus number variation in the two populations of $R$. omeimontis may be a consequence of this underestimation.

\section{Genetic diversity}

The MHC class I sequences determined in both species displayed a comparatively level of high genetic diversity similar to other anuran species (Table 2). Exon 2 and the corresponding domain $\alpha 1$ had the highest diversity in both species, while exon 4 and domain $\alpha 3$ had the lowest. This mutation distribution pattern has been observed in other amphibians [44,72] and can be explained by the distinct functions of the different domains. We also noted that there was a lower level of divergence among nucleotide sequences compared with that among amino acid chains. This observation suggests that there were more non-synonymous compared with synonymous mutations, which is consistent with the observations for the sequences under positive selection.

\section{Detection of recombination}

Using the RDP program, we identified five alleles as recombination products (Table 3). Because we established very conservative parameters to avoid miscalculations, the

Table 1 Summary of population allele distributions

\begin{tabular}{llll}
\hline & $\begin{array}{l}\text { P. megacephalus } \\
\left(\mathrm{HN}^{\mathbf{a}}\right)\end{array}$ & $\begin{array}{l}\text { R. omeimontis } \\
\left(\mathrm{HN}^{\mathbf{a}}\right)\end{array}$ & $\begin{array}{l}\text { R. omeimontis } \\
\left(\mathrm{SC}^{\mathbf{b}}\right)\end{array}$ \\
\hline Sample size & 11 & 12 & 15 \\
$\begin{array}{l}\text { No. of alleles } \\
\begin{array}{l}\text { Maximal No. of } \\
\text { alleles observed } \\
\text { in one sample }\end{array}\end{array}$ & 7 & 6 & 14 \\
$\begin{array}{l}\text { Minimal No. of } \\
\text { loci }\end{array}$ & 2 & 3 & 6 \\
\hline
\end{tabular}

Notes: ${ }^{\text {a } H N ~ H u n a n,}{ }^{\text {b }}$ SC Sichuan. 
Table 2 Average nucleotide and amino acid distances among the 27 newly isolated alleles

\begin{tabular}{lll}
\hline & $\boldsymbol{P}$. megacephalus & $\boldsymbol{R}$. omeimontis \\
\hline K2P nucleotide distance & $0.156(0.010)$ & $0.143(0.009)$ \\
Exon 2 & $0.341(0.034)$ & $0.319(0.031)$ \\
Exon 3 & $0.115(0.014)$ & $0.132(0.014)$ \\
Exon 4 & $0.069(0.011)$ & $0.029(0.006)$ \\
Poisson-corrected amino acid & $0.243(0.023)$ & $0.229(0.022)$ \\
distance & & \\
a1 & $0.486(0.062)$ & $0.452(0.056)$ \\
a2 & $0.213(0.037)$ & $0.240(0.036)$ \\
a3 & $0.099(0.026)$ & $0.053(0.015)$ \\
\hline
\end{tabular}

Notes: Standard errors are shown in parentheses.

actual number of recombinants was probably greater than five. However, the five alleles still accounted for a relatively large proportion (18.5\%) of these alleles. Using the RDP and GARD programs, four $(239,141,334$, and 229) and three $(223,425$, and 531$)$ breakpoints were detected, respectively. Breakpoints located near the boundary of exons 2 and 3 (239, 229, and 223) were found out several times, lending strong support to the presence of recombination via exon shuffling with breakpoints in intron 2 . The same type of recombination has been observed in other frogs and bony fishes $[25,37,44]$.

\section{Detection of selection}

We used three different codon-based maximum likelihood methods all with partitioning pre-analyses to screen for selection signals. Analyses using PAML (Additional files 1 and 2), FEL (Additional file 3), and MEME (Additional file 4) all suggested that Rhacophoridae MHC class Ia alleles underwent natural selection, although the positive selected sites identified by different methods varied (Figure 2 and Table 4). However, the majority of these sites were located on exons 2 and 3 regardless of their differences. In total, 12 out of the 28 sites were identified by more than one program to have undergone positive selection. Excluding two sites on exon 4 and one site on exon 3, the other 9 sites all belonged to the presumed ABS [47,48] (Figure 2). This finding indicated that the Rhacophoridae MHC class I genes underwent pathogen-mediated balancing selection [19].

\section{Phylogenetic analyses}

Phylogenetic trees were constructed using available anuran MHC class I genes. Considering that the exon exchanges occurred between exons 2 and $3 / 4$ and that mutation rates were very different between exons $2 / 3$ and 4 , we partitioned the aligned sequences into individual exon regions to reconstruct more accurate phylogenetic relationships. In all three trees, the MHC class Ia genes clustered together while remaining distinct from the MHC class Ib genes; the 27 newly obtained alleles clearly belonged to the MHC class Ia gene cluster (Figure 3). The tree constructed for exon 4 was very consistent with species tree [42] because there were five clusters that corresponded well to the anuran taxa: the newly obtained alleles from $P$. megacephalus and $R$. omeimontis formed the Rhacophoridae cluster with high confidence; alleles from $L$. yavapaiensis, $L$. clamitans, $L$. catesbeianus, and R. pipiens formed the Ranidae cluster as the sister clade of Rhacophoridae; alleles from S. phaeota and from A. callidryas belonged to the Hylidae cluster and then coalesced with alleles from E. prosoblepon; alleles from Xenopus formed a well-supported clade (Figure 3). In conclusion, exon 4 of the MHC class I genes displayed speciesspecific clustering. However, in the phylogenetic trees of exons 2 and 3, alleles from the same species did not always display more similarity than those from different taxa. In the tree of exon 2, a portion of the Rhacophoridae alleles branched from common ancestors of Rhacophoridae, Ranidae, Hylidae, and Centrolenidae species; the other portion bifurcated into two clusters. In the tree of exon 3, mixing of alleles from different species could be observed too, although the tree exhibited a star-like topology, likely due to the lack of informative mutations (Figure 3). In conclusion, exons 2 and 3 of Rhacophoridae MHC class I genes possess trans-species polymorphisms [29].

\section{Discussion}

We successfully isolated MHC class I genes from two Rhacophoridae species: P. megacephalus and $R$. omeimontis. Several lines of evidence suggest that all of the newly isolated alleles belong to classical MHC class I loci: (1) the alleles were amplified from total RNA instead of DNA, and therefore, pseudogenes were excluded because they cannot be expressed; (2) neither additional stop codons nor abnormal indels were observed in the alleles; (3) putative amino acids forming disulfide bonds were conserved in all of the

Table 3 Recombination test using the RDP program

\begin{tabular}{|c|c|c|c|c|}
\hline & Recombinant sequence & Nucleotide breakpoint & Potential parental sequences & Methods \\
\hline 1 & Rhom16 & 239 & Rhom13/Unknown(Rhom17) & GENECONV, BootScan, MaxChi, Chimaera, Siscan, 3Seq \\
\hline 2 & Pome06 & 239 & Rhom15/Rhom18 & GENECONV, BootScan, MaxChi, Chimaera, Siscan, 3Seq \\
\hline 3 & Rhom17 & 141 & Rhom12/Unknown(Rhom05) & GENECONV, MaxChi, Chimaera, Siscan, 3Seq \\
\hline 4 & Pome04 & 334 & Rhom10/Unknown(Rhom12) & MaxChi, Chimaera, Siscan, 3 Seq \\
\hline 5 & Rhom08 & 229 & Rhom15/Rhom18 & RDP, GENECONV, BootScan, MaxChi, Chimaera, Siscan, $3 \mathrm{Seq}$ \\
\hline
\end{tabular}




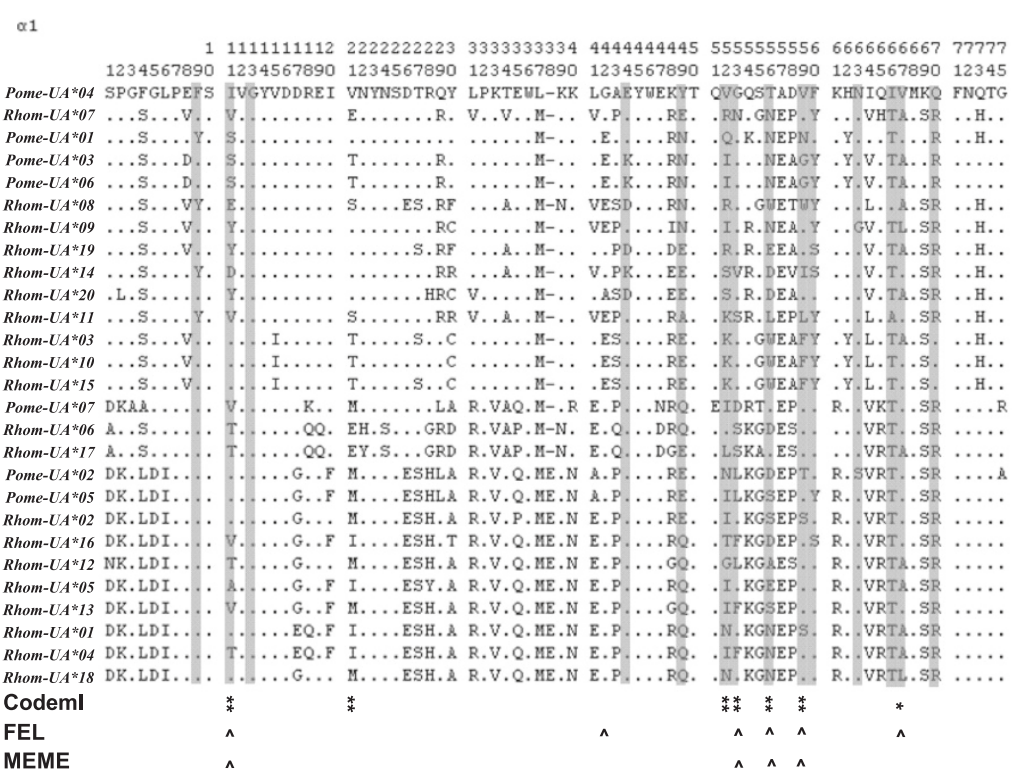

$\alpha 2$

$111111111122222222223 \quad 333333333444444444455555555556 \quad 6666666667 \quad 7777777778 \quad 8888888889999$

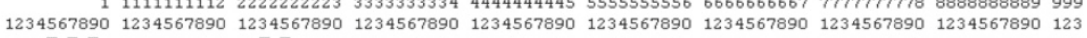
Pome-UA*04 GIHTYQRMTG CELRDDGSTA GYQOIGYDGR DFLYLDTQSU LYIPTMDEAQ LSAQTINGPD VRGGERUKNY LQDICIPULK KYIEYGREDL EKR

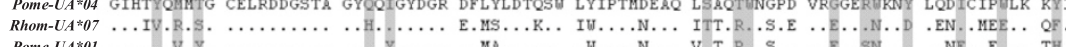

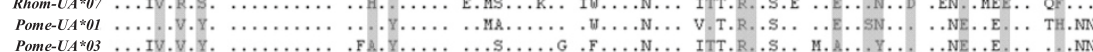

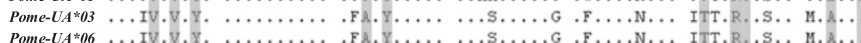

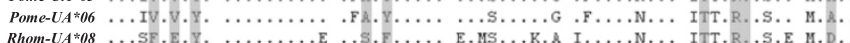

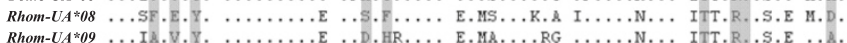

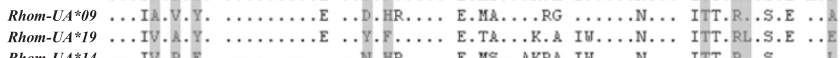

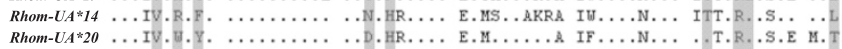

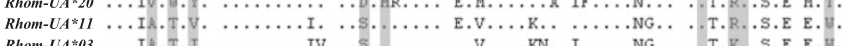

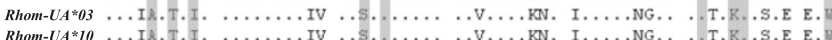



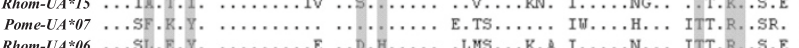

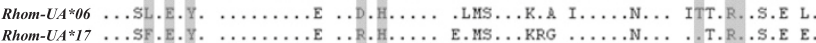

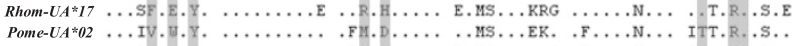

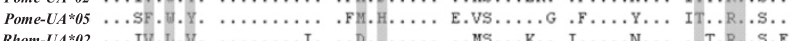

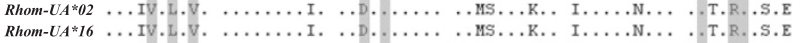


Rhom-UA*05 $\ldots$ SL
Rhom-UA*13 $\ldots$ IV.W.

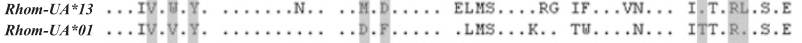
Rhom-UA*01 $\ldots . T V . V$
Rhom-UA*04 $\ldots$ SF.

CodemI $* *$

MEME A $\wedge$ ^
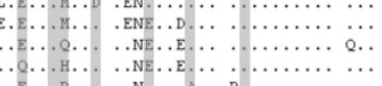
. 
Table 4 Summary statistics for codon sites undergoing positive selection identified by different methods

\begin{tabular}{|c|c|c|c|c|c|c|c|c|c|c|c|c|c|c|c|c|}
\hline Method & Site & redic & to $b$ & nder & sitive & ectio & & & & & & & & & & \\
\hline \multicolumn{17}{|l|}{ a 1} \\
\hline & 11 & 21 & 42 & 52 & 53 & 56 & 59 & 67 & & & & & & & & \\
\hline Codeml $^{a}$ & $* *$ & $* *$ & & $* *$ & $* *$ & ** & ** & * & & & & & & & & \\
\hline $\mathrm{FEL}^{\mathrm{b}}$ & $\wedge$ & & $\wedge$ & & $\wedge$ & $\wedge$ & $\wedge$ & $\wedge$ & & & & & & & & \\
\hline $\mathrm{MEME}^{\mathrm{C}}$ & $\wedge$ & & & & $\wedge$ & $\wedge$ & $\wedge$ & & & & & & & & & \\
\hline \multicolumn{17}{|l|}{$a_{2}$} \\
\hline & 4 & 5 & 7 & 9 & 20 & 23 & 25 & 41 & 42 & 52 & 61 & 63 & 67 & 74 & 77 & 81 \\
\hline Codeml ${ }^{a}$ & & $* *$ & $* *$ & & & $* *$ & $* *$ & & $* *$ & & & $* *$ & $* *$ & & & \\
\hline $\mathrm{FEL}^{\mathrm{b}}$ & & & $\wedge$ & $\wedge$ & $\wedge$ & & & $\wedge$ & $\wedge$ & $\wedge$ & $\wedge$ & $\wedge$ & & & & \\
\hline $\mathrm{MEME}^{\mathrm{C}}$ & $\wedge$ & & $\wedge$ & $\wedge$ & & $\wedge$ & & & & & & & & $\wedge$ & $\wedge$ & $\wedge$ \\
\hline \multicolumn{17}{|l|}{ a 3} \\
\hline & 10 & 34 & 40 & 72 & & & & & & & & & & & & \\
\hline Codeml $\left.\right|^{a}$ & * & $*$ & $* *$ & $* *$ & & & & & & & & & & & & \\
\hline $\mathrm{FEL}^{\mathrm{b}}$ & & & $\wedge$ & $\wedge$ & & & & & & & & & & & & \\
\hline $\mathrm{MEME}^{\mathrm{C}}$ & & & & & & & & & & & & & & & & \\
\hline
\end{tabular}

Notes: Numbers correspond to the alignment shown in Figure 2. ${ }^{a}$ Amino acids identified in model M8 by the Bayes empirical Bayes procedure [67] using Codeml. Only those sites predicted to be undergoing positive selection with a posterior probability $>95 \%$ are presented in the table. The * indicates that the posterior probability is $>95 \%$, and ${ }^{* *}$ shows that the probability is $>99 \%$. ${ }^{b}$ Amino acid sites identified by FEL are identified with $\wedge$. ${ }^{\mathrm{c} A m i n o}$ acid sites identified by MEME are identified with $\wedge$.

one MHC class Ia locus. However, all other vertebrates, including fishes [25], reptiles [79], birds [26] and mammals [12], commonly express more than one locus; a recent study on anuran MHC evolution also revealed that six non-model anuran species all possessed at least two or three MHC class Ia loci [44]. Thus our present study showed two additional anuran species with multiple MHC class Ia loci, supported results of the recent study on anuran MHC class I genes, and presented the ubiquity of multiple MHC class I loci in vertebrates. Our knowledge on amphibian MHC class I genes is updated and broadened by studying more representative species.

High degree of loci number variation has been observed in many vertebrates. For example, Atlantic salmons express three MHC class I loci [80], Chinese sturgeon and paddlefish express two loci [25], while cichlid fishes express 17 loci [81]; chickens have two loci [82] while blue tits express at least 6 loci [26]. For amphibians, previous studies revealed a similar but simple pattern that Anura express one locus $[5,39,40,72,78]$ while Caudata express more than one locus [36]. Based on this limited information, researchers speculated that the diversification of amphibian MHC class I loci numbers occurred sometime after the divergence of Caudata/Anura but before that of Ranidae/Pipidae [40]. However, the present study showed that the numbers of MHC class I loci also varied among the anuran species, which is consistent with one recent study [44]. Number variation of amphibian MHC class I loci is then likely to be more complicated than we thought. After combining all the available information on amphibian MHC class I loci (Figure 4), we found that species belonging to the same family could also have different amounts of loci, suggesting that anuran loci number variation is species specific. This pattern of mixed loci number can be explained by the occurrence of gene duplication/ loss during the evolution of amphibian MHC class I genes, that is, the birth-and-death model of evolution [35]. However, this pattern also can be caused by inaccurate estimations of loci number in each species. The two causes are not incompatible. Further analyses to examine accurate number of anuran MHC class Ia loci can help to understand the complete history of anuran gene duplications.

Interestingly, we even observed loci number variation in two geographic populations of the same species $(R$. omeimontis) (Table 1). However, the observation either mirrors actual loci number variation or just results from underestimation of loci number. We try to distinguish the two causes based on the experiences that MHC alleles from the same locus tended to cluster together $[83,84]$. Phylogenetic tree constructed from exon 2 sequences of anuran MHC class I alleles (Figure 3a) was chosen as reference because this tree had a clear topology due to high polymorphic sequences and had high veracity due to the partitioning strategy. We found that 20 alleles from $R$. omeimontis formed three distant clusters. Alleles both from Hunan population (RhomUA*01 to Rhom-UA*06) and from Sichuan population (from Rhom-UA*07 to Rhom-UA*20) distributed in the three clusters. This pattern suggests that the two 
populations of $R$. omeimontis both have three MHC class I loci, and the observed loci number variation is caused by underestimation of loci number in Hunan population. Previous studies have also revealed locus number variations among individuals or populations of the same species $[13,85]$. Similarly, it is early to take the variations as truth before further analyses were performed to preclude the possibility of loci number underestimation.

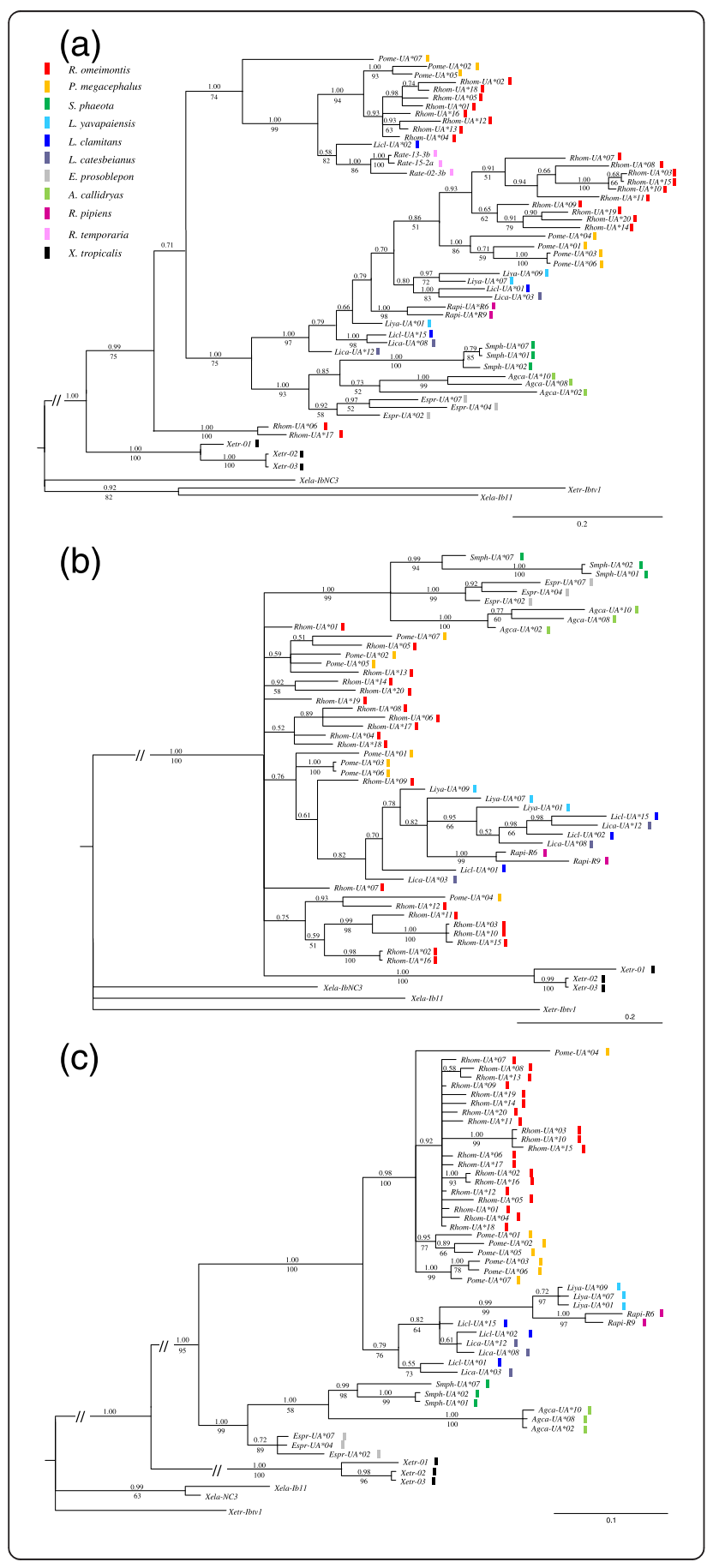

Figure 3 Phylogenetic relationships of exon 2 (a), exon 3 (b), and exon 4 (c) in anuran MHC class I alleles. All of the trees were rooted from MHC Ib genes of Xenopus. Bootstrap values $>50 \%$ (from the neighbor-joining analysis) are shown above the respective clades. Bayesian posterior probabilities $>0.70$ are presented below the clades. All of the branches are proportional to the scale shown at the bottom right of the figure, excluding branches with the sign "//", which were shortened for graphical clarity of the remaining branches of the tree. Alleles belonging to different species are marked using different colored bars. Involved allelic sequences obtained from NCBI include the following: MHC class la alleles from S. phaeota (Smph-UA*07: JQ679386; Smph-UA*02: JQ679381; Smph-UA*01: JQ679380); L. yavapaiensis (Liya-UA*09: JQ679379; Liya-UA*07: JQ679377; Liya-UA*01: JQ679371); L. clamitans (Licl-UA*15: JQ679369; LiCl-UA*02: JQ679356; Licl-UA*01: JQ679355); L. catesbeianus (Lica-UA*12: JQ679354; Lica-UA*08: JQ679350; Lica-UA*03: JQ679345); E. prosoblepon (Espr-UA*07: JQ679337; Espr-UA*04: JQ679334; Espr-UA*02: JQ679332); A. callidryas (Agca-UA*10: JQ679321; Agca-UA*08: JQ679319; Agca-UA*02: JQ679313); R. pipiens (Rapi-R6: AF185587; Rapi-R9: AF185588); R. temporaria (Rate-13-3b: FJ385632; Rate-02-3b: FJ385633; Rate-15-2a: FJ385641);

X. tropicalis (Xetr-01: BC161748; Xetr-02: NM_001113065; Xetr-03: BC154904); and MHC Ib alleles from X. tropicalis (Xetr-lbtv1: NM_001247995) and from X. laevis (Xela-lb11: FJ589643; Xela-lbNC3: L20726).

Recombination is one of the most important factors driving evolution of MHC class I genes [23-26]. In the present study, recombination was found to play an important role in the evolution of Rhacophoridae MHC class Ia genes. $18.5 \%$ of the alleles were generated by recombination, and the percentage is likely an underestimation of the actual value because the alleles were identified using very conservative criteria. Several breakpoints were identified, and a large portion of them were located near the boundary of exons 2 and 3. This finding indicates that the exchange of DNA occurred mainly between exons 2 and 3/4. The recombination pattern is congruent with that mainly observed in the MHC class I genes of bony fishes and other anurans [24,25,37], but different from observations in mammals which have reticulate recombination pattern [34]. It suggests that the MHC class I gene of anuran and of teleostean might have some similar gene organization contributing to the same exon shuffling pattern. Previous studies have revealed that intron 2 of MHC class I genes in bony fishes exhibits very long coverage and contains numerous repeated elements [33]. The two characters of gene organization were regarded to be pivotal to make intron 2 a recombination hot spot region. However, the intron 2 sequences of Rhacophoridae MHC class I genes were not available for analysis. We deduce that Rhacophoridae species likely also have a large intron 2 region in their MHC class I genes based on the genetic information obtained from the whole genome of $X$. tropicalis [37]. Certainly, additional studies specifically on MHC gene organization are required if we want to confirm this deduction.

Several evidence suggests that Rhacophoridae MHC class I genes have undergone natural selection. First, the nucleotide sequence divergence of these genes was reduced compared with the amino acid sequence 


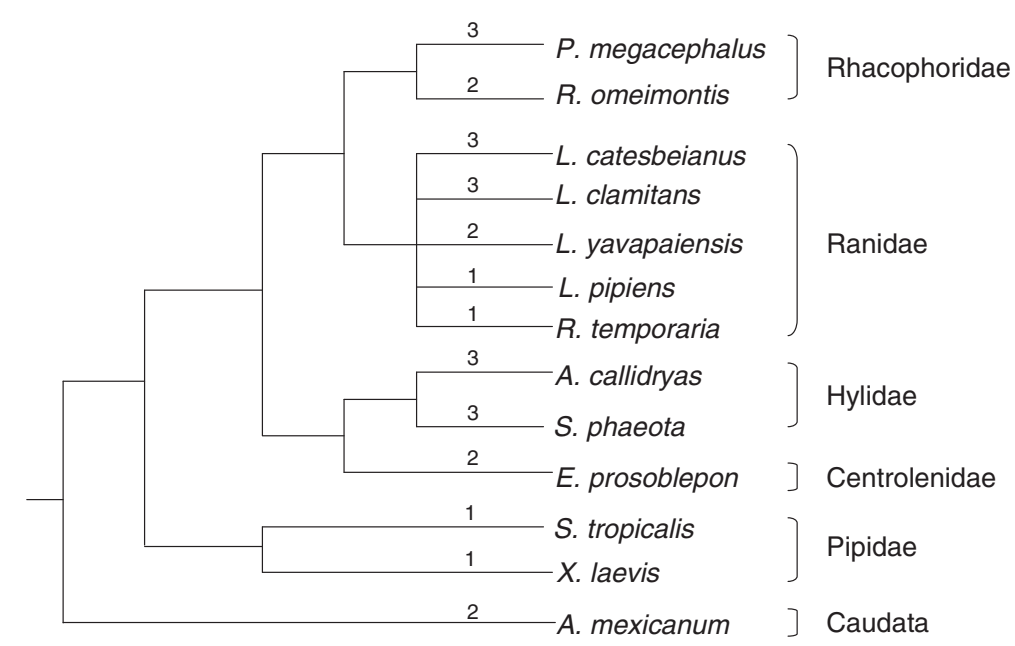

Figure 4 Minimum numbers of putative MHC class la loci in amphibian species. The simplified phylogenetic tree of amphibian species with a deduced number of MHC class la loci is based on the amphibian pedigree shown in [42]. The putative number of loci is shown above the corresponding branches.

divergence, consistent with the positive selection signal showing that non-synonymous were more frequent than synonymous variations. Second, several codonbased methods have detected natural selection signals as well as the sites probably under positive selection in the $27 \mathrm{MHC}$ class I alleles. The sites that were identified by more than one method were considered robust; they belonged to the subset of putative ABS deduced from the crystal structure of human HLA $[47,48]$, with three exceptions. This result indicated that the structures of Rhacophoridae class I MHC molecules were similar to that of human HLA despite some differences. Similar results have been observed in many other species $[25,38]$. Considering the function of ABS, we conclude that the MHC class I genes identified herein are most likely undergoing pathogen-mediated balancing selection.

Trans-species polymorphisms of anuran MHC class Ia genes were evident considering that MHC class Ia alleles from Ranidae, Racophoridae, Hylidae, and Centrolenidae all shared common ancestry (Figure 1). More specifically, the trans-species polymorphisms were observed in exons 2 and 3 of anuran MHC class I genes (Figure 3). When constructing $\mathrm{NJ}$ trees using synonymous mutations, we did not observe species specific topology in the phylogenetic trees of exons 2 and 3 (data not shown). This finding precludes the possibility that concerted evolution caused the trans-species polymorphism. Then balancing selection was most likely to be correlated with the trans-species polymorphism pattern. Specifically speaking, $\alpha 1$ and $\alpha 2$, which contain key regions responsible for antigen recognition and binding, undergo strong balancing selection such that the nucleotide sequences encoding these domains can be maintained for long periods. With sufficient persistence, the origin of the alleles predates the species divergence. In contrast, exon 4 encoding $\alpha 3$, which functions as an anchor and a linker, suffers much fewer selection constraints. Consequently, the pedigree of exon 4 commonly reflects accurate phylogenetic relationships of these species. The difference in evolution patterns for different exons of the same gene has been observed in previous studies $[25,44,84]$.

In trans-species polymorphisms, mixing of alleles from different species results from the long-time persistence of MHC genes which originate before births of the corresponding species [29]. So we can deduce the time when $\mathrm{MHC}$ genes come into being according to divergence time of related species. In the present study, two anuran MHC class Ia allelic lineages were likely to originate respectively before divergence of Ranidae/Racophoridae and that of Hylidae/Centrolenidae (Figure 3a). As these two divergence events respectively date back to 69-72 and 68-91 million years ago (MYA) [43], the oldest MHC class Ia allelic lineages for Anura was estimated to originate about 70 MYA. This time to maintain MHC class I alleles for Anura is considerably long compared to that for some other vertebrates [86]. For example, the oldest allelic lineages at human HLA loci A, B and C was deduced to diverge about 18, 22 and 10 MYA, respectively [29].

\section{Conclusion}

In the present study, we designed new primers to successfully isolate MHC class Ia genes from two Rhacophoridae species ( $P$. megacephalus and $R$. omeimontis). Both species were presumed to contain two or more MHC class Ia locus. Frequent gene duplications, recombination mainly due to exchanges of whole exons, and balancing selection predominantly acting on the ABS, were all considered to 
play an important role in the evolution of Rhacophoridae $\mathrm{MHC}$ class I genes. The current results broaden our knowledge of the evolution of anuran MHC genes.

\section{Additional files}

\begin{abstract}
Additional file 1: Goodness of fit for different codon evolution models and estimated parameter values. Notes: $P$ refers to the number of parameters in the $x$ distribution; $x$ is the selection parameter. $\mathrm{pn}$ is the proportion of sites falling within the $\mathrm{xn}$ site class. For models $\mathrm{M} 7$ and $\mathrm{M} 8, \mathrm{p}$ and $\mathrm{q}$ denote the shape parameters of the $\mathrm{b}$ function. Positively selected sites were identified in models M2a and M8 using the Bayes empirical Bayes procedure [67]. Sites inferred to be undergoing positive selection at the $95 \%$ and $99 \%$ confidence interval level are marked with * and ${ }^{* *}$, respectively.
\end{abstract}

Additional file 2: Summary of the test statistics for the likelihood ratio test of codon evolution. Notes: df refers to degrees of freedom. Test statistics were computed using formula 2 ( $\mathrm{Lb}-\mathrm{La}$ ); La and Lb are log-likelihood values for each of the nested models being compared.

\section{Additional file 3: Sites undergoing positive selection detected} using the FEL program. Note: Numbering of the three domains differs from that shown in Additional file 1. Rather, the sites positions of domains $a 2$ and $a 3$ are counted from the first amino acids of domain a1. Only those sites with a significance level $<0.05$ are shown. aNormalized dN-dS refers to $\mathrm{dN}$-dS divided by the total length of the appropriate tree.

Additional file 4: Sites undergoing positive selection detected using the MEME program. Note: The numbering strategy is described in Additional file 3. This summary table reports the distribution of synonymous ( $a$ ) and non-synonymous ( $\beta$ ) substitution rates for sites inferred using the MEME model. Sites with a significance level $<0.01$ are shown.

\section{Abbreviations}

MHC: Major histocompatibility complex; cDNA: Complementary DNA; PCR: Polymerase chain reaction; ABS: Antigen binding sites; MYA: Million years ago.

\section{Competing interests}

The authors declare no competing interests.

\section{Authors' contributions}

MZ performed the data analysis and drafted the manuscript; YW, HS and CC carried out the molecular experiments; $C L$ performed collection of samples; ZL participated in the data analysis; HW designed the study, participated in the data analysis and helped to draft the manuscript. All authors read and approved the final manuscript.

\section{Acknowledgements}

This work was financially supported by National Natural Science Foundation of China (No.31071934 and 31270425).

\section{Received: 31 December 2012 Accepted: 29 May 2013}

Published: 5 June 2013

\section{References}

1. Klein J: Natural history of the major histocompatibility complex. New York: John Wiley \& Sons; 1986

2. Hughes AL, Yeager M: Natural selection at major histocompatibility complex loci of vertebrates. Annu Rev Genet 1998, 32:415-435.

3. Bodmer W: Evolutionary significance of the HL-A system. Nature 1972, 237:139-183.

4. Piertney SB, Oliver MK: The evolutionary ecology of the major histocompatibility complex. Heredity 2006, 96:7-21.

5. Goyos A, Sowa J, Ohta Y, Robert J: Remarkable conservation of distinct nonclassical MHC class I lineages in divergent amphibian species. $\mathrm{J}$ Immunol 2011, 186:372-381.
6. Flajnik MF, Kasahara M, Shum BP, Salter-Cid L, Taylor E, Du Pasquier L: A novel type of class I gene organization in vertebrates: a large family of non-MHC-linked class I genes is expressed at the RNA level in the amphibian Xenopus. EMBO J 1993, 12:4385-4396.

7. Gaudieri S, Dawkins RL, Habara K, Kulski JK, Gojobori T: SNP profile within the human major histocompatibility complex reveals an extreme and interrupted level of nucleotide diversity. Genome Res 2000, 10:1579-1586.

8. Meyer D, Thomson G: How selection shapes variation of the human major histocompatibility complex: a review. Ann Hum Genet 2001, 65:1-26.

9. Pavelko KD, Mendez-Fernandez Y, Bell MP, Hansen MJ, Johnson AJ, David CS, Rodriguez M, Pease LR: Nonequivalence of classical MHC class I loci in ability to direct effective antiviral immunity. PLoS Pathog 2012, 8:e1002541.

10. Kelley J, Walter $L$, Trowsdale J: Comparative genomics of major histocompatibility complexes. Immunogenetics 2005, 56:683-695.

11. Nonaka M, Namikawa C, Kato Y, Sasaki M, Salter-Cid L, Flajnik MF: Major histocompatibility complex gene mapping in the amphibian Xenopus implies a primordial organization. Proc Natl Acad Sci U S A 1997, 94:5789-5791.

12. Vincek V, Nizetic D, Golubic M, Figueroa F, Nevo E, Klein J: Evolutionary expansion of Mhc class I loci in the mole-rat, Spalax ehrenbergi. Mol Biol Evol 1987, 4:483-491

13. Figueroa F, Mayer WE, Sato A, Zaleska-Rutczynska Z, Hess B, Tichy H, Klein J: Mhc class I genes of swordtail fishes, Xiphophorus: variation in the number of loci and existence of ancient gene families. Immunogenetics 2001, 53:695-708.

14. Horton R, Wilming L, Rand V, Lovering RC, Bruford EA, Khodiyar VK, Lush MJ, Povey S, Talbot CC Jr, Wright MW, et al: Gene map of the extended human MHC. Nat Rev Genet 2004, 5:889-899.

15. Alcaide M, Lemus JA, Blanco G, Tella JL, Serrano D, Negro JJ, Rodriguez A, Garcia-Montijano M: MHC diversity and differential exposure to pathogens in kestrels (Aves: Falconidae). Mol Ecol 2010, 19:691-705.

16. May S, Zeisset I, Beebee TJC: Larval fitness and immunogenetic diversity in chytrid-infected and uninfected natterjack toad (Bufo calamita) populations. Conserv Genet 2011, 12:805-811.

17. Sommer $\mathrm{S}$ : The importance of immune gene variability (MHC) in evolutionary ecology and conservation. Front Zool 2005, 2:16.

18. Bernatchez $L$, Landry C: MHC studies in nonmodel vertebrates: what have we learned about natural selection in 15 years? J Evol Biol 2003, 16:363-377.

19. Spurgin $L G$, Richardson DS: How pathogens drive genetic diversity: MHC, mechanisms and misunderstandings. P Roy Soc B Biol Sci 2010, 277:979-988.

20. Garrigan D, Hedrick PW: Perspective: detecting adaptive molecular polymorphism: lessons from the MHC. Evolution 2003, 57:1707-1722.

21. McCairns RJ, Bourget S, Bernatchez L: Putative causes and consequences of $\mathrm{MHC}$ variation within and between locally adapted stickleback demes. Mol Ecol 2011, 20:486-502.

22. Aguilar A, Roemer G, Debenham S, Binns M, Garcelon D, Wayne RK: High MHC diversity maintained by balancing selection in an otherwise genetically monomorphic mammal. Proc Natl Acad Sci U S A 2004, 101:3490-3494.

23. Jakobsen IB, Wilson SR, Easteal S: Patterns of reticulate evolution for the classical class I and II HLA loci. Immunogenetics 1998, 48:312-323.

24. Consuegra S, Megens HJ, Schaschl H, Leon K, Stet RJ, Jordan WC: Rapid evolution of the $\mathrm{MH}$ class I locus results in different allelic compositions in recently diverged populations of Atlantic salmon. Mol Biol Evol 2005, 22:1095-1106

25. Wang D, Zhong L, Wei Q, Gan X, He S: Evolution of MHC class I genes in two ancient fish, paddlefish (Polyodon spathula) and Chinese sturgeon (Acipenser sinensis). FEBS Lett 2010, 584:3331-3339.

26. Wutzler R, Foerster $K$, Kempenaers B: MHC class I variation in a natural blue tit population (Cyanistes caeruleus). Genetica 2012, 140:349-364.

27. $\mathrm{Xu}$ TJ, Sun YN, Chen SL: Allelic variation, balancing selection and positive selected sites detected from MHC class I alpha gene of olive flounder. Genetica 2010, 138:1251-1259.

28. Adams EJ, Cooper S, Thomson G, Parham P: Common chimpanzees have greater diversity than humans at two of the three highly polymorphic MHC class I genes. Immunogenetics 2000, 51:410-424.

29. Klein J, Sato A, Nagl S, O'hUigín C: Molecular trans-species polymorphism. Annu Rev Ecol Syst 1998, 29:1-21.

30. Klein J: Origin of major histocompatibility complex polymorphism: the trans-species hypothesis. Hum Immunol 1987, 19:155-162.

31. Holmes N, Parham P: Exon shuffling in vivo can generate novel HLA class I molecules. EMBO J 1985, 4:2849-2854. 
32. Miller KM, Li S, Ming TJ, Kaukinen KH, Schulze AD: The salmonid MHC class I: more ancient loci uncovered. Immunogenetics 2006, 58:571-589.

33. Shum BP, Guethlein L, Flodin LR, Adkison MA, Hedrick RP, Nehring RB, Stet RJ, Secombes C, Parham P: Modes of salmonid MHC class I and II evolution differ from the primate paradigm. J Immuno/ 2001, 166:3297-3308.

34. Hughes AL, Hughes MK, Watkins DI: Contrasting roles of interallelic recombination at the HLA-A and HLA-B loci. Genetics 1993, 133:669-680.

35. Nei M, Gu X, Sitnikova T: Evolution by the birth-and-death process in multigene families of the vertebrate immune system. Proc Natl Acad Sci U S A 1997, 94:7799-7806.

36. Sammut B, Du Pasquier L, Ducoroy P, Laurens V, Marcuz A, Tournefier A: Axolotl MHC architecture and polymorphism. Eur J Immunol 1999, 29:2897-2907.

37. Bos DH, Waldman B: Evolution by recombination and transspecies polymorphism in the MHC class I gene of Xenopus laevis. Mol Biol Evol 2006, 23:137-143.

38. Bos DH, Waldman B: Polymorphism, natural selection, and structural modeling of class la MHC in the African clawed frog (Xenopus laevis). Immunogenetics 2006, 58:433-442.

39. Ohta Y, Goetz W, Hossain MZ, Nonaka M, Flajnik MF: Ancestral organization of the MHC revealed in the amphibian Xenopus. J Immunol 2006, 176:3674-3685.

40. Teacher AG, Garner TW, Nichols RA: Evidence for directional selection at a novel major histocompatibility class I marker in wild common frogs (Rana temporaria) exposed to a viral pathogen (Ranavirus). PLoS One 2009, 4:e4616.

41. Shum BP, Avila D, Du Pasquier L, Kasahara M, Flajnik MF: Isolation of a classical MHC class I cDNA from an amphibian. Evidence for only one class I locus in the Xenopus MHC. J Immunol 1993, 151:5376-5386.

42. Roelants K, Gower DJ, Wilkinson M, Loader SP, Biju SD, Guillaume K, Moriau $L$, Bossuyt F: Global patterns of diversification in the history of modern amphibians. Proc Natl Acad Sci U S A 2007, 104:887-892.

43. Roelants K, Haas A, Bossuyt F: Anuran radiations and the evolution of tadpole morphospace. Proc Natl Acad Sci U S A 2011, 108:8731-8736.

44. Kiemnec-Tyburczy KM, Richmond JQ, Savage AE, Lips KR, Zamudio KR: Genetic diversity of MHC class I loci in six non-model frogs is shaped by positive selection and gene duplication. Heredity 2012, 109:146-155.

45. Rozen S, Skaletsky H: Primer3 on the WWW for general users and for biologist programmers. Methods Mol Biol 2000, 132:365-386.

46. Tamura K, Peterson D, Peterson N, Stecher G, Nei M, Kumar S: MEGA5: molecular evolutionary genetics analysis using maximum likelihood, evolutionary distance, and maximum parsimony methods. Mol Biol Evol 2011, 28:2731-2739.

47. Bjorkman PJ, Saper MA, Samraoui B, Bennett WS, Strominger JL, Wiley DC: Structure of the human class I histocompatibility antigen, HLA-A2. Nature 1987, 329:506-512.

48. Bjorkman PJ, Saper MA, Samraoui B, Bennett WS, Strominger JL, Wiley DC: The foreign antigen binding site and $T$ cell recognition regions of class I histocompatibility antigens. Nature 1987, 329:512-518.

49. Pond SLK, Posada D, Gravenor MB, Woelk CH, Frost SD: Automated phylogenetic detection of recombination using a genetic algorithm. $\mathrm{Mol}$ Biol Evol 2006, 23:1891-1901.

50. Martin D, Rybicki E: RDP: detection of recombination amongst aligned sequences. Bioinformatics 2000, 16:562-563.

51. Martin DP, Posada D, Crandall KA, Williamson C: A modified bootscan algorithm for automated identification of recombinant sequences and recombination breakpoints. AIDS Res Hum Retroviruses 2005, 21:98-102.

52. Salminen MO, Carr JK, Burke DS, McCutchan FE: Identification of breakpoints in intergenotypic recombinants of HIV type 1 by bootscanning. AlDS Res Hum Retroviruses 1995, 11:1423-1425.

53. Padidam M, Sawyer S, Fauquet CM: Possible emergence of new geminiviruses by frequent recombination. Virology 1999, 265:218-225.

54. Smith JM: Analyzing the mosaic structure of genes. J Mol Evol 1992, 34:126-129.

55. Posada D, Crandall KA: Evaluation of methods for detecting recombination from DNA sequences: computer simulations. Proc Natl Acad Sci U S A 2001, 98:13757-13762.

56. Gibbs MJ, Armstrong JS, Gibbs AJ: Sister-scanning: a Monte Carlo procedure for assessing signals in recombinant sequences. Bioinformatics 2000, 16:573-582.

57. Boni MF, Posada D, Feldman MW: An exact nonparametric method for inferring mosaic structure in sequence triplets. Genetics 2007, 176:1035-1047.
58. Heath L, van der Walt E, Varsani A, Martin DP: Recombination patterns in aphthoviruses mirror those found in other picornaviruses. J Virol 2006, 80:11827-11832.

59. Guindon S, Gascuel O: A simple, fast, and accurate algorithm to estimate large phylogenies by maximum likelihood. Syst Biol 2003, 52:696-704.

60. Posada D: jModelTest: phylogenetic model averaging. Mol Biol Evol 2008, 25:1253-1256

61. Ronquist F, Teslenko M, van der Mark P, Ayres DL, Darling A, Hohna S, Larget B, Liu L, Suchard MA, Huelsenbeck JP: MrBayes 3.2: efficient Bayesian phylogenetic inference and model choice across a large model space. Syst Biol 2012, 61:539-542.

62. Scheffler $\mathrm{K}$, Martin DP, Seoighe C: Robust inference of positive selection from recombining coding sequences. Bioinformatics 2006, 22:2493-2499.

63. Yang Z: PAML: a program package for phylogenetic analysis by maximum likelihood. Comput Appl Biosci 1997, 13:555-556.

64. Yang Z: PAML 4: phylogenetic analysis by maximum likelihood. $\mathrm{Mol}$ Biol Evol 2007, 24:1586-1591.

65. Goldman N, Yang Z: A codon-based model of nucleotide substitution for protein-coding DNA sequences. Mol Biol Evol 1994, 11:725-736.

66. Yang Z, Nielsen R, Goldman N, Pedersen AM: Codon-substitution models for heterogeneous selection pressure at amino acid sites. Genetics 2000, 155:431-449.

67. Yang Z, Wong WS, Nielsen R: Bayes empirical bayes inference of amino acid sites under positive selection. Mol Biol Evol 2005, 22:1107-1118.

68. Anisimova $M$, Nielsen $R$, Yang Z: Effect of recombination on the accuracy of the likelihood method for detecting positive selection at amino acid sites. Genetics 2003, 164:1229-1236.

69. Pond SLK, Frost SD: Not so different after all: a comparison of methods for detecting amino acid sites under selection. Mol Biol Evol 2005, 22:1208-1222.

70. Murrell B, Wertheim JO, Moola S, Weighill T, Scheffler K, SL Kosakovsky Pond: Detecting individual sites subject to episodic diversifying selection. PLoS Genet 2012, 8:e1002764.

71. Pond SL, Frost SD: A genetic algorithm approach to detecting lineagespecific variation in selection pressure. Mol Biol Evol 2005, 22:478-485.

72. Flajnik MF, Ohta Y, Greenberg AS, Salter-Cid L, Carrizosa A, Du Pasquier L, Kasahara M: Two ancient allelic lineages at the single classical class I locus in the Xenopus MHC. J Immunol 1999, 163:3826.

73. Klein SL, Strausberg RL, Wagner L, Pontius J, Clifton SW, Richardson P: Genetic and genomic tools for Xenopus research: The NIH Xenopus initiative. Dev Dyn 2002, 225:384-391.

74. Ohta Y, Powis SJ, Lohr RL, Nonaka M, Pasquier LD, Flajnik MF: Two highly divergent ancient allelic lineages of the transporter associated with antigen processing (TAP) gene in Xenopus: further evidence for co-evolution among MHC class I region genes. Eur J Immunol 2003, 33:3017-3027.

75. Goyos A, Ohta Y, Guselnikov S, Robert J: Novel nonclassical MHC class lb genes associated with CD8 T cell development and thymic tumors. Mol Immunol 2009, 46:1775-1786.

76. Li SS, Hu JS: Studies on the cytogentics of four Rhacophorid tree frogs from Yunnan. Zool Res 1996, 17:483-488.

77. Rao DQ, Yang DT: Studies on karyotypes of eight species of Rhacophoridae in Yunnan. Sichuan J Zool 1996, 15:105-109.

78. Flajnik MF, Ohta Y, Namikawa-Yamada C, Nonaka M: Insight into the primordial MHC from studies in ectothermic vertebrates. Immunol Rev 1999, 167:59-67.

79. Miller HC, Belov K, Daugherty CH: MHC Class I genes in the Tuatara (Sphenodon spp.): evolution of the MHC in an ancient reptilian order. Mol Biol Evol 2006, 23:949-956.

80. Lukacs MF, Harstad H, Bakke HG, Beetz-Sargent M, McKinnel L, Lubienieck KP, Koop BF, Grimholt U: Comprehensive analysis of MHC class I genes from the U-, S-, and Z-lineages in Atlantic salmon. BMC Genomics 2010, 11:154.

81. Sato A, Klein D, Sultmann H, Figueroa F, O'HUigin C, Klein J: Class I mhc genes of cichlid fishes: identification, expression, and polymorphism. Immunogenetics 1997, 46:63-72

82. Kaufman J, Milne S, Gobel TW, Walker BA, Jacob JP, Auffray C, Zoorob R, Beck S: The chicken $B$ locus is a minimal essential major histocompatibility complex. Nature 1999, 401:923-925.

83. Babik W, Pabijan M, Radwan J: Contrasting patterns of variation in MHC loci in the Alpine newt. Mol Ecol 2008, 17:2339-2355. 
84. Nonaka Ml, Aizawa K, Mitani H, Bannai HP, Nonaka M: Retained orthologous relationships of the MHC Class I genes during euteleost evolution. Mol Biol Evol 2011, 28:3099-3112.

85. Xu S, Ren W, Zhou X, Zhou K, Yang G: Sequence polymorphism and geographical variation at a positively selected MHC-DRB gene in the finless porpoise (Neophocaena phocaenoides): implication for recent differentiation of the Yangtze finless porpoise? J Mol Evol 2010, 71:6-22.

86. Piontkivska $\mathrm{H}$, Nei M: Birth-and-death evolution in primate MHC class I genes: divergence time estimates. Mol Biol Evol 2003, 20:601-609.

doi:10.1186/1471-2148-13-113

Cite this article as: Zhao et al.: Evolution by selection, recombination, and gene duplication in MHC class I genes of two Rhacophoridae species. BMC Evolutionary Biology 2013 13:113.

\section{Submit your next manuscript to BioMed Central and take full advantage of:}

- Convenient online submission

- Thorough peer review

- No space constraints or color figure charges

- Immediate publication on acceptance

- Inclusion in PubMed, CAS, Scopus and Google Scholar

- Research which is freely available for redistribution 